\title{
The Effect of Inhibitors on the Electron-transport Chain of Bacillus brevis. Evidence for Branching of the NADH Oxidase Respiratory Chain
}

\author{
By B. SEDDON* AND JEAN MCVITTIE \\ Department of Biochemistry, University of Liverpool, P.O. Box 147 , \\ Liverpool L69 $3 B X$
}

(Received I August I973; revised 30 May 1974)

Previous inhibitor studies on the electron-transport system of Bacillus brevis ATCC 10068 with NADH as substrate showed the incomplete inhibition of NADH oxidase by cyanide and antimycin A (Seddon \& Fynn, 197I ; Fynn \& Seddon, 1972). The effects of tyrocidine on component enzymes of the electron-transport chain and of culture age on NADH oxidase have also been reported (Seddon \& Fynn, 1973). We now present data showing that component enzymes in the electron-transport particles can be completely inhibited by cyanide and antimycin $\mathbf{A}$ and that tyrocidine and tyrothricin cause inhibition of NADH oxidase at or near the level of NADH dehydrogenase, whereas succinoxidase and cytochrome $c$ oxidase activities are considerably less sensitive to the inhibitors. In line with this observation there is no decrease in activity of succinoxidase throughout growth, but NADH oxidase activity falls dramatically.

\section{METHODS}

Organism, growth and maintenance. Bacillus brevis ATCC 10068 was maintained and grown as described by Fynn, Thomas \& Seddon (1972) on the asparagine--glycerol, basalsalts medium used by Mach, Reich \& Tatum (1963). Bacteria, unless otherwise stated, were harvested during exponential growth by centrifuging at $3000 \mathrm{~g}$ for $20 \mathrm{~min}$. Growth was followed by measuring the increase in turbidity at $600 \mathrm{~nm}$.

Preparation of electron-transport particles (e.t.p.). These were obtained by the method of Seddon \& Fynn (197I).

Enzyme assays. NADH oxidase, NADH dehydrogenase (ferricyanide), NADH-menadione reductase, NADH-cytochrome $c$ reductase, cytochrome $c$ oxidase activities and the effects of $\mathrm{KCN}$, antimycin A, tyrocidine and tyrothricin were measured with the e.t.p. preparation as detailed by Fynn \& Seddon (1972). The method of Smith \& Camerino (I963) was used in the assay of cytochrome $c$ oxidase. Coritrols without added cytochrome $c$ or minus enzyme preparation gave only slight oxidation of ascorbate. The correction for autoxidation of ascorbate never exceeded $10 \%$ and in most cases was much less than this. Duplicate determinations of oxidase activity agreed to within $7 \%$. Succinate oxidation was measured polarographically (Seddon \& Fynn, 197I).

Protein determination. Protein concentrations were measured by the procedure of Lowry, Rosebrough, Farr \& Randall (195I) using crystalline bovine plasma albumin (fraction V) as standard.

* Present address: Department of Developmental Biology, University of Aberdeen, Marischal College, Aberdeen AB9 IAS. 
Chemicals. The chemicals used throughout this work were of analytical reagent quality when available. More specialized chemicals were obtained from Sigma, except bovine plasma albumin (fraction V), and tyrothricin and tyrocidine, which were obtained from Armour Pharmaceutical Co. Ltd, Eastbourne, Sussex, and Nutritional Biochemicals Co. Ltd, Cleveland, Ohio, respectively.

\section{RESULTS AND DISCUSSION}

NADH oxidase activity varies with culture age (Seddon \& Fynn, 1973). In order to minimize the effects of endogenous tyrothricin production the electron-transport particles were prepared from organisms freshly harvested from the exponential phase of growth, before substantial amounts of tyrothricin were produced. Because of variations in NADH oxidase activity between individual preparations all component enzyme activities and inhibitor effects were measured on the same preparation. Thus direct comparisons of the component enzyme activities with the overall NADH oxidase activity could be made. Table I shows results obtained from a typical preparation: (For each component enzyme activity and inhibitor effect at least three experiments were made. The inhibitions obtained consistently gave values within $5 \%$ of those shown.)

Complete inhibition of NADH oxidation with $\mathrm{KCN}$ was never obtained. Approximately 15 to $20 \%$ of the NADH oxidase activity remained with a concentration of KCN that gave $100 \%$ inhibition of cytochrome $c$ oxidase. The high concentration of $\mathrm{KCN}$ had no effect on NADH dehydrogenase activity, indicating that $\mathrm{KCN}$ was effectively inhibiting cytochrome oxidase only. The activity of NADH-cytochrome $c$ reductase, however, when no $\mathrm{KCN}$ was present, was only $2 \%$ of the activity observed when $\mathrm{KCN}$ was present to block the action of cytochrome oxidase. These results suggest that the rate-limiting step in the overall NADH oxidase sequence is between components of the NADH-cytochrome $c$ reductase system that follow those involved in the NADH-ferricyanide dehydrogenase reaction. Hence $\mathrm{KCN}$ is necessary when measuring $\mathrm{NADH}-$ cytochrome $c$ reductase activity.

The partial enzyme activity rates except for that of NADH-cytochrome $c$ reductase which is only approximately $80 \%$ of the overall activity, are equal to, or greater than, the overall NADH oxidase rate. This value of $80 \%$ may possibly be a result of mammalian cytochrome $c$ (an artificial redox component in this sytem) being the electron acceptor. On the other hand mammalian cytochrome $c$ reacts favourably in the cytochrome $c$ oxidase system, which suggests that mammalian cytochrome $c$ is able to interact at the locus of $B$. brevis cytochrome $c$. There could be a more reasonable explanation of these findings. If NADH-cytochrome $c$ reductase is the rate-limiting step, then one must infer that NADH-cytochrome $c$ reductase participation can account for only $80 \%$ of the overall rate of NADH oxidation. This agrees with the fact that only approximately $80 \%$ of the NADH oxidase activity is inhibited by $\mathrm{KCN}$, whereas cytochrome $c$ oxidase is $100 \%$ inhibited. The remaining $20 \%$ of the NADH oxidase activity would be via a pathway from NADH to oxygen which does not pass through the $\mathrm{KCN}$-sensitive cytochrome oxidase.

As with $\mathrm{KCN}$, maximum inhibition with antimycin A accounts for only about $80 \%$ of the overall NADH oxidase activity (Table I), whereas inhibition of the partial electrontransfer region containing the antimycin A-sensitive site (NADH-cytochrome $c$ reductase) causes complete loss of activity. The total electron flow cannot, therefore, pass through the antimycin A-sensitive site and so the residual 15 to $20 \%$ NADH oxidase activity is via an electron-transfer pathway not involving the inhibitor-sensitive site. The NADH dehydrogenase region is not affected by antimycin $\mathrm{A}$, so the site of inhibition must be after the 
participation of ferricyanide but before the participation of cytochrome $c$. The characteristics of inhibition are similar to those observed with mitochondrial systems and the inhibitory site could be around the level of cytochrome $b$.

The results with $\mathrm{KCN}$ indicate a branching in the respiratory chain at a point on the dehydrogenase side of cytochrome oxidase. Similarly, the results with antimycin A suggest that this branch point is at a level on the flavoprotein side of the antimycin A-sensitive site (from the present experiments located between the dehydrogenase and cytochrome $c$ ). Previous observations of incomplete inhibition of NADH oxidation are, therefore, not a result of the relative insensitivity of the inhibitory site but a characteristic brought about by branching of the electron-transport chain. Branched electron-transport occurs in other bacterial systems (White \& Sinclair, 197I). The pattern of antimycin A and KCN inhibition of the individual enzyme components suggests that NADH-cytochrome $c$ reductase and cytochrome $c$ oxidase belong to a common pathway. In contrast NADH-cytochrome $c$ reductase, $\mathrm{NADH}$-menadione reductase and $\mathrm{NADH}$-ferricyanide reductase activities are all inhibited by concentrations of tyrocidine or tyrothricin comparable to those needed for I $00 \%$ inhibition of NADH oxidase (Table I) suggesting that the branch-point lies between NADH and the quinone participant. The level of tyrocidine or tyrothricin inhibition with the NADH-linked oxidation must occur prior to any merging of pathways with succinate oxidation, otherwise succinate oxidation would be inhibited to the same extent as NADH oxidation (see below). Although the results are not unequivocal, the following electrontransport sequence for the NADH oxidase chain of $B$. brevis is proposed from the results presented here and in earlier reports (Fynn, 1973; Fynn et al. 1972; Seddon, 197 ; Seddon \& Fynn, 1971):

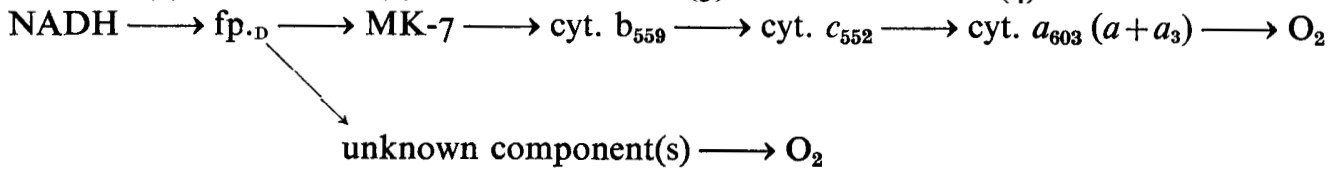

where $\mathrm{fp}_{\cdot \mathrm{D}}$ is NAD-associated flavoprotein dehydrogenase, MK-7 is menaquinone-7, cyt. is cytochrome, and (I), (2), (3) and (4) indicate primary sites of inhibition by tyrothricin, u.v. irradiation, antimycin $\mathrm{A}$ and $\mathrm{KCN}$ respectively. The residual activity after $\mathrm{KCN}$ and antimycin $\mathbf{A}$ inhibition or u.v. irradiation is presumably via the unidentified pathway. Tyrocidine inhibition, however, must occur prior to the branch-point at the level of NADH dehydrogenase, since $100 \%$ inhibition of NADH oxidase and NADH dehydrogenase was observed.

Table I also shows the inhibition by the cyclic decapeptide antibiotics of NADH oxidase (and its component enzyme systems), succinoxidase and cytochrome $c$ oxidase activities. Complete inhibition of succinoxidase activity was obtained only with concentrations of tyrocidine and tyrothricin threefold higher than those required for $100 \%$ inhibition of NADH oxidase. Cytochrome $c$ oxidase was not affected by the antibiotics at the levels which produced $100 \%$ inhibition of NADH oxidase activity, and an eightfold increase in antibiotic concentration was required for substantial inhibition $(80 \%)$ of activity. The results indicate that NADH oxidation is preferentially inhibited.

Electron-transport particles isolated from various stages of growth showed that NADH oxidase activity declined from 145 to $22 \mathrm{ng}$-atom $\mathrm{O}_{2} / \mathrm{min} / \mathrm{mg}$ protein as growth increased; succinoxidase activity remained at a low but constant value (about $\mathrm{I} 2 \mathrm{ng}$-atoms $\mathrm{O}_{2} / \mathrm{min} / \mathrm{mg}$ protein). Increased tyrothricin production at later stages of growth (Seddon \& Fynn, I973) may affect the primary NADH dehydrogenase site, but the tyrothricin cannot be present 
Table I. Activities of electron-transport component enzymes and the effects of inhibitors

\begin{tabular}{|c|c|c|c|c|}
\hline Enzyme component & $\begin{array}{l}\text { Inhibitor } \\
\text { concentration } \\
(\mu \mathrm{mol} / \mathrm{mg} \\
\text { protein })\end{array}$ & $\begin{array}{l}\text { Specific } \\
\text { activity* }\end{array}$ & $\begin{array}{l}\text { Relative } \\
\text { activity } \dagger\end{array}$ & $\begin{array}{c}\text { Inhibition } \\
(\%)\end{array}$ \\
\hline \multicolumn{5}{|l|}{ NADH oxidase } \\
\hline Alone & 0.0 & 103 & 100 & 0 \\
\hline$+\mathbf{K C N}$ & $1 \cdot 0$ & 52 & 50 & 50 \\
\hline$+\mathrm{KCN}$ & $13 \cdot 0$ & 18 & 17 & $83 \div$ \\
\hline + Antimycin A & $1 \cdot 3$ & 16 & 16 & 84 \\
\hline + Tyrocidine & 0.4 & 0 & 0 & 100 \\
\hline + Tyrothricin & 0.6 & 0 & 0 & 100 \\
\hline \multicolumn{5}{|c|}{ Cytochrome $c$ oxidase } \\
\hline Alone & 0.0 & $40 I$ & 390 & 0 \\
\hline$+\mathrm{KCN}$ & $13 \cdot 0$ & 0 & 0 & 100 \\
\hline +Antimycin A & $1 \cdot 3$ & 400 & 388 & 0 \\
\hline + Tyrocidine & $3 \cdot 3$ & 80 & 78 & $80 \ddagger$ \\
\hline + Tyrothricin & $5 \cdot 5$ & 82 & 80 & $81 \ddagger$ \\
\hline \multicolumn{5}{|c|}{ NADH dehydrogenase } \\
\hline Alone & 0.0 & 155 & 150 & 0 \\
\hline$+\mathrm{KCN}$ & 13.0 & 158 & 151 & 0 \\
\hline + Antimycin A & $I \cdot 3-5 \cdot 5$ & I 53 & 149 & 0 \\
\hline + Tyrocidine & 0.4 & 0 & 0 & 100 \\
\hline + Tyrothricin & 0.6 & o & o & 100 \\
\hline \multicolumn{5}{|c|}{ NADH-cytochrome $c$ reductase $\S$} \\
\hline Alone & 0.0 & 80 & 78 & 0 \\
\hline +Antimycin A & $1 \cdot 3$ & 0 & 0 & 100 \\
\hline + Tyrocidine & 0.4 & 0 & 0 & 100 \\
\hline + Tyrothricin & 0.6 & o & o & 100 \\
\hline \multicolumn{5}{|c|}{ NADH-menadione reductase } \\
\hline Alone & 0.0 & 620 & 602 & 0 \\
\hline + Tyrocidine & 0.4 & o & o & 100 \\
\hline + Tyrothricin & 0.6 & o & o & 100 \\
\hline \multicolumn{5}{|l|}{ Succinoxidase } \\
\hline Alone & 0.0 & 12 & I 2 & 0 \\
\hline + Tyrocidine & $1 \cdot 5$ & 0 & 0 & 100 \\
\hline + Tyrothricin & $1 \cdot 9$ & 0 & 0 & 100 \\
\hline
\end{tabular}

* Quoted as nmol substrate converted/min/mg protein.

$\dagger$ Relative to the NADH oxidase activity as $100 \%$.

$\$$ Maximum inhibition observed; higher concentrations produced no further inhibition of activity.

$\S \mathrm{KCN}$ already present (see details of assay system).

in high enough concentrations to inhibit succinate oxidation to any extent. The possibility cannot be disregarded, however, that reduced oxygen tension during late growth may have influenced the NADH oxidase (Seddon \& Fynn, 1973).

The results here indicate that NADH, succinate and cytochrome $c$ oxidases all show different levels of inhibition with the cyclic decapeptide antibiotics. The antibiotics are presumed to act as cationic detergents (Hunter \& Schwartz, 1967) and it would appear that different levels of membrane disorganization lead to the results observed. It is suggested that the three levels of antibiotic inhibition of enzyme activity are due to the different environments of NADH dehydrogenase, succinate dehydrogenation and cytochrome $c$ oxidase within the membrane lipoprotein matrix. Low levels of cyclic decapeptides are required to disrupt the NADH dehydrogenase region whilst, at the other extreme, only with high levels of antibiotic is there observable inhibition of cytochrome $c$ oxidase. 


\section{REFERENCES}

FynN, G. H. (1973). Menaquinone function in the electron transport system of an antibiotic-producing strain of Bacillus brevis. Journal of General Microbiology 74, 181-184.

Fynn, G. H. \& Seddon, B. (1972). Terminal oxidations in Bacillus brevis. Soluble reduced nicotinamide adenine dinucleotide oxidase activity in Bacillus brevis. Journal of General Microbiology 73, 189-192.

FynN, G. H., Thomas, D. V. \& SEDdon, B. (1972). On the role of menaquinone in the reduced nicotinamide adenine dinucleotide oxidative pathway of Bacillus brevis. Journal of General Microbiology 70, $271-275$.

Hunter, F. E. \& SchWARTz, L. S. (1967). In Antibiotics, vol, 1, pp. 636, 643. Edited by D. Gottlieb and P. D. Shaw. New York: Springer-Verlag.

Lowry, O. H., Rosebrough, N. J., Farr, A. L. \& Randall, R. J. (I95I). Protein measurement with the Folin phenol reagent. Journal of Biological Chemistry 193, 265-275.

Mach, B., Reich, E. \& TAtum, E. L. (1963). Separation of the biosynthesis of the antibiotic polypeptide tyrocidine from protein biosynthesis. Proceedings of the National Academy of Sciences of the United States of America 50, I75-181.

SEDDON, B., (1971). Studies concerning the respiratory metabolism of a tyrothricin producing strain of Bacillus brevis. Ph.D. thesis, University of Manchester, Institute of Science and Technology.

Seddon, B. \& FYNN, G. H. (I97I). Terminal oxidations in Bacillus brevis. II. The electron-transport system of Bacillus brevis. Archiv für Mikrobiologie 77, 252-261.

SEDdon, B. \& FYNN, G. H. (1973). Energetics of growth in a tyrothricin-producing strain of Bacillus brevis. Journal of General Microbiology 74, 305-314.

SMith, L. \& CAMERINo, P. W. (1963). Comparison of polarographic and spectrophotometric assays for cytochrome $c$ oxidase activity. Biochemistry 2, 1428-1432.

Whíte, D. C. \& SinclaiR, P. R. (I97I). Branched electron-transport systems in bacteria. In Advances in Microbial Physiology, pp. 173-208. Edited by A. H. Rose and J. F. Wilkinson. New York: Academic Press. 